\title{
BIOMASSA E MACRONUTRIENTES DE UMA FLORESTA ESTACIONAL DECIDUAL EM ITAARA-RS, BRASIL ${ }^{1}$
}

\author{
Hamilton Luiz Munari Vogel², Mauro Valdir Schumacher ${ }^{3}$ e Peter Trüby ${ }^{4}$
}

\begin{abstract}
RESUMO - Este trabalho foi realizado no Município de Itaara-RS, Brasil, em uma Floresta Estacional Decidual secundária. Teve como objetivo quantificar os nutrientes na biomassa acima do solo, de essências florestais. Foram abatidas 20 árvores, distribuídas em cinco classes de diâmetros $(3,2-13,5 \mathrm{~cm} ; 13,6-23,9 \mathrm{~cm} ; 24,0-33,6 \mathrm{~cm}$; 33,7-44,6 cm; e 44,7-55,0 cm). A parte aérea foi fracionada em madeira do fuste, casca do fuste, galhos e folhas. Nas amostras da biomassa foram determinados os teores de N, P, K, Ca, Mg e S. O estoque de nutrientes na biomassa de cada componente foi obtido com base na biomassa seca estimada, multiplicada pelo teor de nutrientes. O rendimento total de biomassa foi estimado em $210,0 \mathrm{Mg} \mathrm{ha}^{-1}$. Para esse rendimento, as contribuições percentuais dos galhos, madeira do fuste, casca do fuste e folhas foram de 48,8; 43,3; 5,4; e 2,4, respectivamente. As folhas foram o componente com os maiores teores de $\mathrm{N}, \mathrm{P}, \mathrm{K}, \mathrm{Mg}$ e S, enquanto no componente casca se observou o maior teor de $\mathrm{Ca}$. A madeira do fuste foi o componente que apresentou os menores teores de nutrientes em sua biomassa. De modo geral, o $\mathrm{Ca}, \mathrm{N}$ e o $\mathrm{K}$ foram os nutrientes com maiores estoques na biomassa acima do solo. Nos galhos, devido à maior biomassa, foram verificados os maiores estoques de nutrientes, seguidos pela madeira do fuste.
\end{abstract}

Palavras-chave: Floresta nativa, Biomassa acima do solo, Nutrientes.

\section{MACRONUTRIENTS QUANTIFICATION IN A SEASONAL DECIDUAL FOREST IN ITAARA-RS, BRAZIL}

\begin{abstract}
The present study was conducted in a fragment of a seasonal deciduous forest in Itaara-RS, Brazil. The objective of this work was to quantify the nutrient in above ground biomass of forestry species. For the above ground biomass evaluation, 20 trees were cut down according to five different diameter classes $(3,2-13,5 \mathrm{~cm} ; 13,6-23,9 \mathrm{~cm} ; 24,0-33,6 \mathrm{~cm} ; 33,7-44,6 \mathrm{~cm} ; 44,7-55,0 \mathrm{~cm})$. The above ground biomass was separated in the compartment trunk wood, trunk bark, branches and leaves. The contents of $N, P, K, C a$, $M g$ and $S$ in the biomass samples were determined. The stock of nutrients in the biomass for each component was obtained based on the estimated dry biomass, multiplied by the nutrient content. The total production of above ground biomass was estimated at $210.0 \mathrm{Mg}$ ha-1. The branches, trunk wood, trunk bark and leaves corresponded to 48.8, 43.3, 5.4 and $2.4 \%$ of the above ground biomass. The largest contents of $N, P, K$, $M g$ and $S$ were verified in leaves; while greatest Ca content was found in trunk bark. The trunk wood was the component that showed the lowest concentration of nutrients in its biomass. In general, $\mathrm{Ca}, \mathrm{N}$ and $\mathrm{K}$ are the nutrients with the largest stock in the above ground biomass. The largest stocks of nutrients are present in branches, which is mainly due to the great biomass, followed by trunk wood.
\end{abstract}

Keywords: Above ground biomass, Native forest, Nutrient.

\footnotetext{
${ }^{1}$ Recebido em 03.09.2009 aceito para publicação em 19.12.2012.

${ }^{2}$ Universidade Federal do Pampa - UNIPAMPA, São Gabriel-RS. E-mail: <hamiltonvogel@ unipampa.edu.br>.

${ }^{3}$ Universidade Federal de Santa Maria - UFSM, Centro de Ciências Rurais, Departamento de Ciências Florestais, Santa Maria-RS, Brasil.E-mail: <mvschumacher@gmail.com>.

${ }^{4}$ Albert-Ludwigs-Universität Freiburg, Bertoldstr, Freiburg-Alemanha. E-mail: <peter.trueby @ bodenkunde.uni-freiburg.de> .
} 


\section{INTRODUÇÃO}

Nas florestas nativas do Rio Grande do Sul até o momento, poucos estudos foram realizados envolvendo a dinâmica de nutrientes, sobretudo, na região de ocorrência da Floresta Estacional Decidual. Nessas regiões é comum a remoção de áreas da floresta para o estabelecimento de cultivos agrícolas, enquanto a fertilidade do solo permita colheitas satisfatórias e, posteriormente, o abandono da agricultura. Com isso, a abertura de novas áreas de floresta levou à formação de um mosaico de diversos estágios sucessionais na paisagem, com diferentes graus de degradação (BRUN et al., 2010).

Em um ecossistema florestal existe, assim, grande dinâmica na produção de biomassa. À medida que a floresta se desenvolve, principalmente em plantações florestais na fase inicial de crescimento, ocorre gradual redução na biomassa da copa das árvores e, simultaneamente, aumento na proporção dos componentes madeira e casca (os troncos das árvores geralmente representam, em média, mais de $80 \%$ da biomassa acima do solo em uma floresta plantada com idade de corte) (SCHUMACHER; HOPPE, 1997).

A acumulação de biomassa é afetada por fatores ambientais, como luz, temperatura, concentração de $\mathrm{CO}_{2}$ do ar, umidade e fertilidade do solo; e doenças e, também, por fatores de cada planta, como idade, estrutura e disposição das folhas, distribuição e comportamento dos estômatos, teor de clorofila, entre outros (KRAMER; KOZLOWSKI, 1972).

Na biomassa acima do solo de uma floresta nativa ou plantada estão estocadas grandes quantidades de nutrientes. De acordo com Landsberg (1986), as reservas de nutrientes nas florestas estão presentes nos vários componentes das árvores, no solo e na vegetação de sub-bosque, estando, do montante, somente pequena fração em fluxo entre os vários compartimentos do sistema.

Nas zonas temperadas, grande porção de material orgânico e de nutrientes disponíveis está sempre situada nos solos, ao passo que nos trópicos porcentagem muito maior está localizada na biomassa e é reciclada rapidamente no interior das florestas (ODUM; BARRETT, 2008).

Em relação à estimativa da biomassa de uma floresta nativa, esta pode variar muito com o tipo de floresta e local onde se encontra. Em Florestas Estacional Decidual, Ombrófila Mista, Tropical Amazônica, Tropicais
Asiáticas e Temperada na Alemanha (Fagus silvatica), as estimativas das biomassas totais foram de $186,25 \mathrm{Mg} \mathrm{ha}^{-1}$ (BRUN, 2004), 210,4 $\mathrm{Mg} \mathrm{ha}^{-1}$ (CALDEIRA, 2003), 324,0 $\mathrm{Mg} \mathrm{ha}^{-1}$ (HIGUCHI et al., 1998), $225 \mathrm{Mg} \mathrm{ha}^{-1}$ (BROWN et al.,1991) e $313 \mathrm{Mg} \mathrm{ha}^{-1}$ (OTTO, 1994), respectivamente.

Dessa maneira, estudos envolvendo quantificação de biomassa constituem a base para o conhecimento do estoque e dinâmica dos nutrientes na floresta e de como manejar esses ecossistemas de forma sustentável, podendo-se destacar a quantificação da ciclagem de nutrientes, a quantificação de biomassa para fins industriais e energéticos e como base de informação para estudos de sequestro de carbono (SILVEIRA et al., 2008), com grande importância para a tomada de decisões no manejo dos recursos florestais. Nesse contexto, o trabalho teve como objetivo quantificar a produção de biomassa acima do solo e nutrientes das árvores em uma Floresta Estacional Decidual em Itaara, RS, Brasil.

\section{MATERIAL E MÉTODOS}

O trabalho foi realizado em uma fazenda da Fundação da Brigada Militar, no Município de Itaara, Estado do Rio Grande do Sul, Brasil. Esse município está localizado na Serra de São Martinho, na região fisiográfica do Planalto Médio. Verifica-se, na Figura 1, a localização

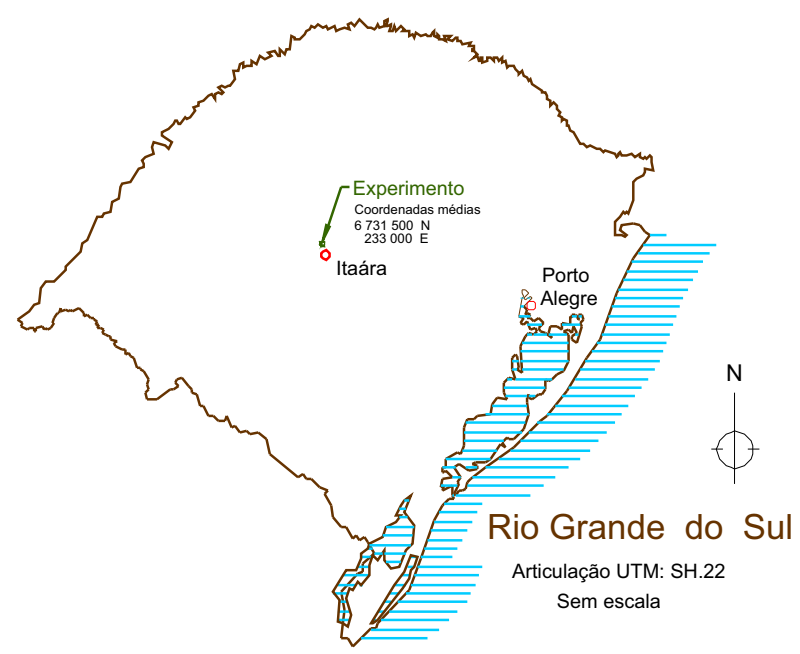

Figura 1 - Localização do Município de Itaara e da área experimental no Estado do Rio Grande do Sul, Brasil.

Figure 1 - Location of the city of Itaara and the experimental area in the State of the Rio Grande do Sul, Brazil.

Revista Árvore, Viçosa-MG, v.37, n.1, p.99-105, 2013 
do Município de Itaara e da área experimental com as coordenadas médias, no Estado do Rio Grande do Sul, sendo $400 \mathrm{~m}$ a altitude média do local.

A região onde o Município de Itaara está inserido apresenta clima Cfa (classificação climática de Köppen). De acordo com a Plataforma de Coleta de Dados do INPE de São Martinho da Serra, RS (estação mais próxima da área experimental), no período compreendido entre 2003 e 2006 , a temperatura média foi de $19,6^{\circ} \mathrm{C}$ e a precipitação anual, de $1.520,5 \mathrm{~mm}$.

O solo da região é classificado como Neossolo Litólico Eutrófico típico (STRECK et al., 2008). Essa classe de solos é descrita pela Embrapa (1999) como "solos com horizonte A ou O hístico com menos de $40 \mathrm{~cm}$ de espessura, assentado diretamente sobre a rocha ou sobre um horizonte $\mathrm{C}$ ou $\mathrm{Cr}$ ou sobre material com $90 \%$ ou mais de sua massa, constituída por fragmentos de rocha com diâmetro superior a $2 \mathrm{~mm}$ (cascalhos, calhaus e matacões)". O solo do local de estudo apresentou profundidade de $35 \mathrm{~cm}$.

Na área estudada, para caracterização da vegetação da Floresta Estacional Decidual foi realizado um inventário, por Longhi et al. (2005), em uma área com aproximadamente 30 ha. Nessa área foram instaladas, sistematicamente, 14 parcelas de $10 \mathrm{~m}$ x $10 \mathrm{~m}$. Nessas parcelas foram amostrados todos os indivíduos com circunferência à altura do peito $(\mathrm{CAP}) \geq 10 \mathrm{~cm}$, os quais foram identificados medindo-se o CAP e a altura total desses indivíduos. Dos indivíduos que não foram passíveis de identificação a campo, coletou-se material botânico, para posterior identificação no Herbário do Departamento de Ciências Florestais CCR-UFSM.

Conforme o inventário publicado por Greff et al. (2006) sobre a composição florística, foram encontradas 52 espécies, divididas em 24 famílias, destacando-se as famílias Leguminosae, Euphorbiaceae e Sapindaceae, em razão de os seus representantes apresentarem os maiores valores do Índice de Valor de Importância IVI (estimadores da importância ecológica de um táxon (espécie, família etc.) dentro de uma comunidade florestal); a família com maior número de representantes foi a Myrtaceae, porém todos eles com baixos valores nos índices relativos; as espécies de maior destaque foram Parapiptadenia rigida e Sebastiana commersoniana devido a seus altos valores no IVI, que totalizaram 48,73\%; a Parapiptadenia rigida apresentou o maior valor de Dominância (área basal de determinada espécie por unidade de área) e a Sebastiana commersoniana, densidade e frequência maiores. Quanto a idade e estágio de desenvolvimento, conforme as informações da Fundação da Brigada Militar, responsável pelo local, estima-se que a floresta tenha em torno de 70 anos de idade e esteja em estágio secundário (LONGHI et al., 2005; GREFF et al., 2006).

De acordo com as informações do inventário, foram determinadas cinco classes de diâmetro, de maneira a abranger a maior variação possível do povoamento. $\mathrm{O}$ intervalo de classe foi obtido pela fórmula $\mathrm{h}=\mathrm{H}$ / $\mathrm{K}$, em que $\mathrm{H}=$ amplitude total (DAP máximo-DAP mínimo) (DAP: diâmetro à altura do peito) e $\mathrm{K}=$ número de classes, com intervalo de classe igual a 10,36. No total, foram abatidas 20 árvores, distribuídas nas classes de diâmetros da seguinte forma: 3,2-13,5 cm com 11 árvores, 13,6-23,9 cm com quatro árvores, 24,0-33,6 cm com três árvores, 33,7-44,6 cm com 1 árvore, 44,7$55,0 \mathrm{~cm}$ com 1 árvore.

Nessa amostragem não foram consideradas as lianas e as árvores mortas. Todas as árvores foram cortadas e cubadas conforme o método de Smalian (descrito por FINGER, 1992), obtendo-se os DAPs e as alturas totais e dos troncos. Após a cubagem, as árvores foram separadas nos componentes madeira do fuste, casca do fuste, galhos e folhas. Estabeleceu-se como madeira do fuste a madeira a partir da base do tronco até a sua primeira bifurcação (conhecida como "forquilha"), sendo, então, o restante do tronco considerado como galhos. Os galhos não foram amostrados por diâmetro, não sendo divididos em galhos grossos e finos, em razão da grande variabilidade morfológica das árvores. Na sequência, cada componente da árvore foi separado e pesado na sua totalidade. Para as amostras de madeira do fuste e casca do fuste, foram retirados três discos do tronco, a $0,10 \mathrm{~cm}$ da altura do fuste, na metade da altura do fuste e no final da altura do fuste. Nesses discos foi separada a casca da madeira, estimando-se, assim, a quantidade de casca de todo o fuste. As coletas de biomassa foram obtidas no período de maio a julho de 2005 .

A estimativa da biomassa de cada componente das árvores de cada uma das parcelas do inventário foi obtida pelo uso da equação $\log \mathrm{y}=\mathrm{b}_{0}+\mathrm{b}_{1} \cdot \log$ DAP (em que $\log \mathrm{y}=\log$ aritmo do componente; $\mathrm{b}_{0}$ e $b_{1}=$ coeficientes da equação; e $\log \mathrm{DAP}=\log$ aritmo do diâmetro da árvore a 1,30 m de altura). A equação utilizada na estimativa foi selecionada em função do 
ajuste satisfatório da estimativa da biomassa acima do solo para os componentes estudados constatado pelos altos valores do coeficiente de determinação ajustados e os baixos erros-padrão e percentual da estimativa (Tabela 1).

Para determinação da umidade e análise química dos nutrientes em laboratório, de cada componente foi retirada uma amostra de aproximadamente $300 \mathrm{~g}$, para obtenção do peso de matéria fresca. Essas amostras foram secas em estufa a $75^{\circ} \mathrm{C}$ até atingir peso constante, obtendo-se o peso de matéria seca. Na sequência, o material foi moído em moinho do tipo Willey, para realização das análises químicas. Foram determinados os teores totais de N, P, K, Ca, Mg e S, segundo a metodologia sugerida por Tedesco et al. (1995) e Miyazawa et al. (1999), da seguinte forma: teores de nitrogênio pelo método Kjeldahl, fósforo por espectrometria visível, potássio por fotometria de chama, enxofre por turbidimetria e cálcio e magnésio por espectrofotometria de absorção atômica. Todas as análises foram realizadas no Laboratório de Ecologia Florestal do Departamento de Ciências Florestais da UFSM. O estoque de nutrientes na biomassa de cada componente foi obtido com base na biomassa seca estimada multiplicada pelo teor de nutrientes do respectivo componente.

\section{RESULTADOS E DISCUSSÃO}

Observa-se, na Tabela 1, que a média da produção total da biomassa acima do solo estimada foi de 210,0 $\mathrm{Mg} \mathrm{ha}^{-1}$. Nessa estimativa, os galhos corresponderam a $48,8 \%$ da biomassa acima do solo, seguidos da madeira do fuste com $43,4 \%$, da casca do fuste com $5,4 \%$ e das folhas com $2,4 \%$.

Nota-se, na Tabela 1, que foi obtido um ajuste satisfatório na estimativa da biomassa acima do solo para os componentes estudados, constatado pelos valores elevados do coeficiente de determinação ajustados ( $R^{2}$ aj.) e pelos baixos erros-padrão percentual da estimativa (Syx). A equação utilizada no trabalho já apresentou boa estimativa da biomassa em florestas plantadas de acácia-negra, eucalipto e pinus (BARICHELLO, 2003), o que também foi verificado neste estudo, dentro das condições observadas.

Em Floresta Estacional Decidual Secundária com cerca de 53 anos de idade, em Santa Tereza, RS, Brun (2004) encontrou biomassa acima do solo de $157,6 \mathrm{Mg} \mathrm{ha}^{-1}$, sendo a maior parte desta formada pela madeira do fuste $(52,41 \%)$, seguido pelos galhos $(28,4 \%)$. Conforme esse autor, nesse estudo os menores valores obtidos dos galhos se deveram, em parte, ao limite estabelecido entre tronco e galhos utilizados, tomados na bifurcação da árvore, podendo gerar alturas maiores, aumentando o comprimento do tronco e diminuindo a proporção relativa de galhos.

Na Floresta Tropical Amazônica, Higuchi et al. (1998) estimaram a biomassa acima do solo em 324,0 Mg ha ${ }^{1}$, com os troncos correspondendo a 65,6\% e a copa a $34,4 \%$, a qual foi dividida em galhos grossos (diâmetro $>1,0 \mathrm{~cm}$ ) com 17,8\%, galhos finos com 14,5\%, folhas com $2,03 \%$ e flores/frutos com $0,01 \%$. Golley et al. (1978), em Floresta Tropical Úmida no Panamá, constataram realidades bastante diferentes, de acordo com o local de amostragem, encontrando biomassa acima do solo de $367,9 \mathrm{Mg} \mathrm{ha}^{-1}$ no sítio da estação úmida e $263,5 \mathrm{Mg} \mathrm{ha}^{-1}$ no sítio da estação seca. No Paraná, em Floresta Ombrófila Mista Montana em General Carneiro, Caldeira (2003) estimou biomassa total acima do solo das árvores com DAP $\geq 10 \mathrm{~cm}$, de $210,4 \mathrm{Mg} \mathrm{ha}^{-1}$, assim distribuída: galhos $(45,3 \%)>$ madeira do fuste $(41,1 \%)>$ casca $(10,3 \%)>$ folhas $(2,4 \%)$. Já na região

Tabela 1 - Coeficientes da equação $\log y=b_{0}+b_{1}(\log$ AP) e estimativa dos componentes da biomassa, acima do solo, da Floresta Estacional Decidual em Itaara-RS, Brasil.

Table 1 - Coefficients of the equation logy $=b_{0}+b_{I}(\log D A P)$ and estimate of the components of above ground biomass of the Seasonal Decidual forest in Itaara-RS, Brazil.

\begin{tabular}{|c|c|c|c|c|c|}
\hline Componente & $\mathrm{b}_{0}$ & $\mathrm{~b}_{1}$ & $\mathrm{R}^{2} \mathrm{aj} \cdot{ }^{1}$ & $\operatorname{Syx}(\%)^{2}$ & Biomassa Estimada $\left(\mathrm{Mg} \mathrm{ha}^{-1}\right)$ \\
\hline Madeira do fuste & $-0,785273$ & 2,029922 & 0,98 & 8,1 & 91,0 \\
\hline Casca do fuste & $-1,547650$ & 1,917776 & 0,94 & 5,0 & 11,4 \\
\hline Galhos & $-1,959650$ & 2,935550 & 0,91 & 8,1 & 102,4 \\
\hline Folhas & $-2,322213$ & 2,250508 & 0,88 & 9,9 & 5,1 \\
\hline Biomassa total & $-0,882390$ & 2,409594 & 0,98 & 8,0 & 210,0 \\
\hline
\end{tabular}

${ }^{1} \mathrm{R}^{2}$ aj.: coeficiente de determinação ajustado; ${ }^{2} \operatorname{Syx}(\%)$ : erro-padrão da estimativa, em percentagem.

Revista Árvore, Viçosa-MG, v.37, n.1, p.99-105, 2013 
de Araucária, PR, também em Floresta Ombrófila Mista, Socher et al. (2008) estimaram biomassa acima do solo de $195 \mathrm{Mg} \mathrm{ha}^{-1}$.

Quanto aos componentes da biomassa, pode-se verificar, na Tabela 2, que a concentração dos nutrientes varia conforme o componente amostrado.

Quanto aos teores de macronutrientes na biomassa, em geral a folha é o componente que apresenta os maiores teores de N, P, K, Mg e S, exceto o Ca. Na casca estão os maiores teores de Ca. O Ca é um elemento considerado imóvel no floema (MALAVOLTA, 1985). A sua imobilidade no floema das plantas pode explicar a sua concentração elevada na fração casca, assim como o fato de o elemento ser componente estrutural, fazendo parte da lamela média da membrana celular (BRUN, 2004).

A madeira do fuste foi o componente que apresentou os menores teores de nutrientes na biomassa acima do solo, em relação aos demais (Tabela 2). Brun (2004) também observou em uma Floresta Estacional Decidual secundária em Santa Tereza, RS, que na madeira do fuste estavam os menores teores de nutrientes.

Marschner (1995) apontou que os teores médios de $15 \mathrm{~g} \mathrm{~kg}^{-1}$ no N, $2 \mathrm{~g} \mathrm{~kg}^{-1}$ no P, $10 \mathrm{~g} \mathrm{~kg}^{-1}$ no K, $5 \mathrm{~g}$ $\mathrm{kg}^{-1}$ no Ca, $2 \mathrm{~g} \mathrm{~kg}^{-1}$ no $\mathrm{Mg}$ e $1 \mathrm{~g} \mathrm{~kg}^{-1}$ no $\mathrm{S}$ estavam dentro das faixas adequadas para plantas superiores.

Em floresta secundária Estacional Decidual em Santa Tereza, RS, Brun (2004) encontrou na biomassa acima do solo teores médios de $17 \mathrm{~g} \mathrm{~kg}^{-1} \mathrm{de} \mathrm{N}, 0,9 \mathrm{~g} \mathrm{~kg}^{-1}$ de P, 12,2 $\mathrm{g} \mathrm{kg}^{-1}$ de $\mathrm{K}, 19,9 \mathrm{~g} \mathrm{~kg}^{-1}$ de Ca, 2,8 $\mathrm{g} \mathrm{kg}^{-1}$ de $\mathrm{Mg}$ e $1,0 \mathrm{~g} \mathrm{~kg}^{-1}$ de $\mathrm{S}$.

Quanto ao estoque de nutrientes, pode-se observar, na Tabela 3, que o componente galhos foi o que mais

Tabela 2 - Teores de macronutrientes na biomassa, acima do solo, nos componentes das árvores, na Floresta Estacional Decidual em Itaara-RS, Brasil.

Table 2 - Contents of nutrients in the above ground biomass components in the trees in Seasonal Decidual forest in Itaara-RS, Brazil.

\begin{tabular}{lrrrrrr}
\hline Componente & $\mathrm{N}$ & $\mathrm{P}$ & $\mathrm{K}$ & $\mathrm{Ca}$ & $\mathrm{Mg}$ & $\mathrm{S}$ \\
\cline { 2 - 7 } & \multicolumn{7}{c}{$\mathrm{g} \mathrm{kg}^{-1}$} \\
\hline Madeira do fuste & 3,1 & 0,2 & 2,6 & 3,7 & 1,2 & 0,9 \\
Casca do fuste & 12,5 & 0,5 & 4,6 & 24,9 & 2,7 & 1,3 \\
Galhos & 9,4 & 0,5 & 5,4 & 12,1 & 2,1 & 1,6 \\
Folhas & 24,2 & 1,2 & 16,6 & 12,4 & 6,4 & 3,4 \\
\hline
\end{tabular}

estocou nutrientes em sua biomassa, com mais de $55 \%$ do N, P, K, Ca, Mg e S, seguido da madeira do fuste. Os galhos e a madeira do fuste, em virtude de sua maior biomassa acumulada, constituíram as principais frações em armazenamento de nutrientes na biomassa acima do solo. Nesse sentido, a ordem do estoque de macronutrientes dos componentes analisados ficou assim estabelecida: galhos $>$ madeira do fuste $>$ casca do fuste $>$ folhas.

O nutriente com maior estoque na biomassa acima do solo foi o $\mathrm{Ca}$, seguido do $\mathrm{N}$, apontando a seguinte ordem crescente de quantidade: $\mathrm{Ca}>\mathrm{N}>\mathrm{K}>\mathrm{Mg}>$ $\mathrm{S}>\mathrm{P}$ (Tabela 3 )

Golley et al. (1978), em Florestas Tropicais no Panamá, verificaram que os elementos mais abundantes na vegetação acima do solo foram o Ca e o K, seguidos do Mg e P (o N não foi considerado neste estudo devido ao método de extração utilizado).

Brun (2004) encontrou estoques de $1.531,5 \mathrm{~kg} \mathrm{ha}^{-1}$ de N, 94,0 kg ha-1 de P, 1.005,7 kg ha-1 de K, 2.443,3 $\mathrm{kg} \mathrm{ha}^{-1} \mathrm{de} \mathrm{Ca}, 288,8 \mathrm{~kg} \mathrm{ha}^{-1} \mathrm{de} \mathrm{Mg}$ e $114,7 \mathrm{~kg} \mathrm{ha}^{-1}$ de $\mathrm{S}$ na biomassa acima do solo, em uma floresta secundária Estacional Decidual em Santa Tereza, RS; pôde-se verificar que os valores encontrados por esse autor estão relativamente próximos dos deste estudo.

Em dois fragmentos florestais montanos da Mata Atlântica (Floresta Ombrófila Densa) na região Norte do Estado do Rio de Janeiro, Cunha et al. (2009) encontraram valor médio de biomassa (parte aérea + serapilheira) de $166,8 \mathrm{Mg} \mathrm{ha}^{-1}$, com estoques médios de N, P, K, Ca e Mg na vegetação de 1.152; 44,4; 276,5; 603,5 ; e $127,9 \mathrm{~kg} \mathrm{ha}^{-1}$, respectivamente.

Em floresta nativa são inúmeros os fatores que afetam a produção de biomassa e a concentração de nutrientes. O material de origem do solo, a profundidade, o clima e a alta biodiversidade das espécies vegetais são alguns dos fatores que mais influem na produção. Também se devem levar em conta as entradas de nutrientes, poeiras e outros elementos provenientes, principalmente, das precipitações, que incidem diretamente na floresta. Na região de estudo eram comuns e frequentes as queimadas de campos nativos no inverno, para renovar a pastagem. Essas queimadas liberam nutrientes por volatilização, os quais retornam para a floresta, em parte com as águas das chuvas.

Revista Árvore, Viçosa-MG, v.37, n.1, 99-105, 2013 
Tabela 3 - Estoque de macronutrientes na biomassa, acima do solo, das árvores e sua participação relativa por componente, na Floresta Estacional Decidual em Itaara-RS, Brasil.

Table 3 - Stock of nutrients in above ground biomass of trees and their relative distribution in per components in Seasonal Decidual forest in Itaara-RS, Brazil.

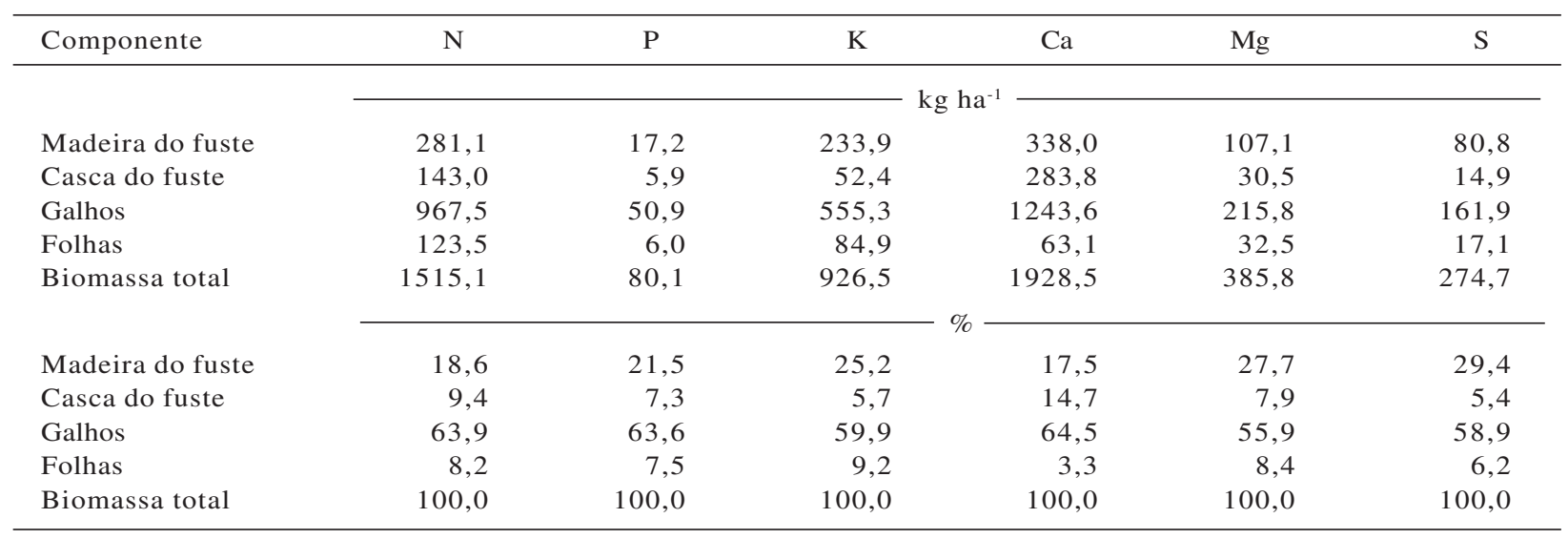

\section{CONCLUSÕES}

1. Na biomassa estimada de $210,0 \mathrm{Mg} \mathrm{ha}^{-1}$, as contribuições percentuais dos galhos, madeira do fuste, casca do fuste e folhas foram de 48,8;43,3; 5,4; e 2,4, respectivamente.

2. Os maiores teores de N, P, K, Mg e S ocorreram nas folhas, e o maior teor de Ca se deu na casca. A biomassa da madeira do fuste apresentou os menores teores de nutrientes.

3. Nos galhos, devido à maior biomassa, estão os maiores estoques de nutrientes, seguidos pela madeira do fuste.

4. De modo geral, o Ca foi o nutriente com maior estoque na biomassa acima do solo, seguido do $\mathrm{N}$ e do K.

\section{AGRADECIMENTOS}

À Capes, pela bolsa de estudos de doutorado no Brasil ao primeiro autor; e ao DAAD, pela bolsa de estudos na Alemanha.

\section{REFERÊNCIAS}

BARICHELLO, L. R. Quantificação da biomassa e dos nutrientes em floresta de Acacia mearnsii De Wild. na região sul do Brasil. 2003. 58f. Dissertação (Mestrado em Silvicultura) - Universidade Federal de Santa Maria, Santa Maria, 2003.

Revista Árvore, Viçosa-MG, v.37, n.1, p.99-105, 2013
BROWN, S.; GILLESPIE, A. J. R.; LUGO, A. E. Biomass of tropical forests of south and Southeast Asia. Canadian Journal of Forest Research, v.21, n. 1, p.111 - 117, 1991.

BRUN, E. J. Biomassa e nutrientes na floresta Estacional Decidual, em Santa Tereza, RS. 2004. 136f.

Dissertação (Mestrado em Silvicultura) Universidade Federal de Santa Maria, Santa Maria, 2004.

BRUN, E. J. et al. Dinâmica de micronutrientes na biomassa florestal em estágios sucessionais de Floresta Estacional Decidual, RS, Brasil.

Scientia Forestalis, v.38, n.86, p.307-318, 2010.

CALDEIRA, M. V. W. Determinação de biomassa e nutrientes em uma floresta Ombrófila Mista Montana em General Carneiro, Paraná. 2003. $176 f$. Tese (Doutorado em Engenharia Florestal) Universidade Federal do Paraná, Curitiba, 2003.

CUNHA, G. M. et al. Biomassa e estoque de carbono e nutrientes em florestas montanas da Mata Atlântica na região norte do estado do Rio de Janeiro. Revista Brasileira de Ciência do Solo, v.33, n.5, p.1175$1185,2009$. 
EMPRESA BRASILEIRA DE PESQUISA AGROPECUÁRIA - EMBRAPA. Centro Nacional de Pesquisa de Solos. Sistema brasileiro de classificação de solos. Rio de Janeiro: 1999. 412p.

FINGER, C. A. G. Fundamentos de biometria florestal. Santa Maria: UFSM/ CEPEF/FATEC, 1992. 269p.

GOlley, F. B. et al. Ciclagem de minerais em um ecossistema de floresta Tropical Úmida. São Paulo: EPU/USP, 1978. 256p.

GREFF, L. T. B. et al. Estrutura fitossociológica de um fragmento de Floresta Estacional Decidual no município de Itaara, RS. In: SIMPÓSIO DE PÓSGRADUAÇÃO EM CIÊNCIAS FLORESTAIS, 4., 2006, Piracicaba. Anais... Piracicaba: ESALQ, 2006. CD ROM.

HIGUCHI, N. et al. Biomassa da parte aérea da vegetação da floresta tropical úmida de terra firme da Amazônia Brasileira. Acta Amazônica, v.28, n.1, p.153-166, 1998.

KRAMER, R. J.; KOSLOWSKI, T. T. Fisiologia das árvores. Lisboa: Fundação Kalouste Gouldbenkian, 1972. 745p.

LANDSberG, J. J. Physiological ecology of forest production. London: Academic Press, 1986. 198p.

LONGHI, S. J. et al. Caracterização fitossociológica de um fragmento de Floresta Estacional Decidual em Itaara,RS. Santa Maria: 2005. Relatório Técnico.
MALAVOLTA, E. Absorção e transporte de íons e nutrição mineral. In: FERRI, M. G. Fisiologia vegetal 1. São Paulo: EPU, 1985. p.77-116.

MARSCHNER, H. Mineral nutrition of higher plants. 2.ed. London: Academic Press, 1995. 889p.

MIYAZAWA, M. et al. Análises químicas de tecido vegetal. In: SILVA, F. C. (Org.). Manual de análises químicas de solos, plantas e fertilizantes. Brasília: Embrapa Comunicação para Transferência de Tecnologia, 1999. p.171-224.

ODUM, E. P. \& BARRETT, G. W.

Fundamentos de ecologia. São Paulo: Cengage Learning, 2008. 612p.

OTTO, H. J. Waldökologie. Stuttgart: Ulmer, 1994. 391p.

SCHUMACHER, M. V.; HOPPE, J. M. A complexidade dos ecossistemas. Porto Alegre: Pallotti, 1997. 50p.

SILVEIRA, P. et al. O estado da arte na estimativa de biomassa e carbono em formações florestais. Revista Floresta, v.38, n.1, p.185-206, 2008.

SOCHER, L. G.; RODERJAN, C. V.; GALVÃO, F. Biomassa aérea de uma foresta ombrófla mista aluvial no município de Araucária (PR). Revista Floresta, v.38, n.2, p.245-252, 2008.

STRECK, E. V. et al. Solos do Rio Grande do Sul. Porto Alegre: EMATER/RS, 2008. 222p.

TEDESCO, M. J. et al. Análise de solo, plantas e outros materiais. Porto Alegre: UFRGS, 1995. 174p. (Boletim técnico, 5). 
